\title{
The efficacy of Bifidobacterium quadruple viable tablet in the treatment of diarrhea predominant irritable bowel syndrome: a randomized, double- blind, placebo-controlled, multicenter trial.
}

\section{Haoyu Zeng}

Wuhan Union Hospital https://orcid.org/0000-0002-9355-4154

\section{Tao Bai}

Wuhan Union Hospital

\section{Yanqin Long}

Sir Run Run Shaw Hospital, Zhejiang University School of Medicine

Xiaoqing Li

Sir Run Run Shaw Hospital, Zhejiang University School of Medicine

\section{Xiaohong Sun}

Peking Union Medical College Hospital

\section{Yu Lan}

Beijing Jishuitan Hospital

\section{Lingling Gao}

Peking University Clinical Research Institute

\section{Lu Zhang}

Hangzhou Grand Biologic Pharmaceutical. INC.

\section{Zenghui Feng}

Hangzhou Grand Biologic Pharmaceutical. INC

Xiaohua Hou ( $\nabla$ houxh@hust.edu.cn )

\section{Study protocol}

Keywords: Irritable bowel syndrome, Diarrhea predominant IBS, Randomized controlled trial, Efficacy, Probiotics

Posted Date: November 18th, 2019

DOI: https://doi.org/10.21203/rs.2.13843/v2

License: (c) (i) This work is licensed under a Creative Commons Attribution 4.0 International License. Read Full License 
Page $2 / 23$ 


\section{Abstract}

Background: Irritable bowel syndrome (IBS) is one of the most acommon functional gastrointestinal disorders characterized by recurrent abdominal pain associated with defecation or a change in bowel habits. Leading to significant negative effect on patients' quality of life and huge financial burden to health system, the management of IBS is a great challenge. Probiotics are considered as an effective therapy, however, in a lack of high-quality evidence of efficacy, no specific strain- and dose-probiotics were recommended in clinical guidelines. This study aims to evaluate the efficacy of the bifidobacterium quadruple viable tablet in the treatment of IBS-D. Methods/design: A multicenter randomized controlled trial will be performed in fourteen hospitals. A total of three hundred patients fulfill the eligible criteria will be stratified divided into an experimental group and a control group randomly in a ratio of 1:1. The experimental group is treated with the bifidobacterium quadruple viable tablet while the control group is treated with placebo. All the patients will receive a 4-week treatment and a 2-week follow-up. The primary outcome is the effectiveness in improving abdominal pain and stool consistency, the secondary outcome include evaluation of overall symptom relief, frequency of defecation, bloating, urgency of defecation, remedial medication, score of IBS-QOL and changes of microbiota and metabonomics. Physical examination, vital signs, laboratory tests, adverse events and concomitant medication will be taken into account for intervention safety assessment during the trial. Discussion: This multicenter randomized controlled trial may provide high-quality evidence on the efficacy of the bifidobacterium quadruple viable tablet for IBS-D on both physical and mental dimensions in China. To fill the gap of previous probiotics intervention studies, in addition, this study will also present safety assessment which will be a significant emphasis.

\section{Background}

Irritable bowel syndrome (IBS) is one of the most common functional gastrointestinal disorders characterized by recurrent abdominal pain associated with defecation or a change in bowel habits ${ }^{[1,2]}$. The prevalence is estimated between $5-20 \%$ worldwide ${ }^{[3]}$. Based on the predominant disorder in bowel habits, IBS is classified into four subtypes: constipation predominant IBS (IBS-C), diarrhea predominant IBS (IBS-D), IBS with mixed bowel habits (IBS-M) and unclassified IBS (IBS-U). Among them, IBS-D is the most common one with a proportion of nearly $60 \%$ in IBS according to a cross-sectional survey ${ }^{[4]}$. Although IBS-D affects patients' quality of life and brings huge financial burden to health system, the management is still a great challenge ${ }^{[5,6]}$.

Nowadays, more and more studies focus on the role of microbiota in patients with IBS ${ }^{[7-12]}$. Recently published studies found that, compared with normal participants, there is a significant reduction in beneficial bacteria such as Bifidobacteria and Lactobacilli. Instead, an increase of Escherichia coli in patients with IBS-D is observed ${ }^{[11,12]}$. Therefore, the change of the microbiota is deemed as an important etiology of IBS-D. 
A newly published systematic review supported the benefits of probiotics for patient with IBS, but there was significant heterogeneity as for the various kind and dose of probiotics ${ }^{[13]}$. Besides, there are very few high quality randomized controlled trials with rigorous design ${ }^{[13,14]}$. As a result, we need further strong evidence to guide us as for the clinical choice of probiotics.

The bifidobacterium quadruple viable tablet is a new candidate of probiotic product developed and produced by Hangzhou Grand Biologic Pharmaceutical. INC (Patent number: ZL01108353.0; International Patent Classification: A61K 35/74) ${ }^{[15]}$. The bifidobacterium quadruple viable tablet is a compound microecological preparation consisting of Bifidobacterium infantis, Lactobacillus acidophilus, Enterococcus faecalis and Bacillus cereus, combining anaerobic with aerobic bacteria. On one hand, Bifidobacterium infantis, Lactobacillus acidophilus and Enterococcus faecalis are normal intestinal microbiota inside intestinal tract of healthy human body. Studies found that direct supplementation of microbiota above can inhibit certain pathogenic bacteria, and leads to normal intestinal peristalsis and balance of intestinal microbiome ${ }^{[16-21]}$. On the other hand, Bacillus cereus can colonize in the intestinal tract. They consume oxygen and create an anaerobic environment for anaerobic bacteria to promote the growth and reproduction of anaerobic bacteria such as bifidobacteria ${ }^{[22]}$. We speculated that the four strains of the bifidobacterium quadruple viable tablet can collaborate to multi-target improvement of the intestinal microenvironment, which indicated a potential therapeutic effect on IBS-D.

In all, the aim of this trial is to evaluate the efficacy of the bifidobacterium quadruple viable tablet in the treatment of IBS-D.

\section{Methods/design}

\section{Trial design}

This study is a parallel-group, double-blind, placebo-controlled, multicenter randomized controlled trial (RCT). The trial will include three periods: a 2-week screening period, a 4-week treatment period, and a 2week follow-up period. During the screening period, patients will record a symptom diary every day including abdominal pain intensity, stool consistency, evaluation of symptoms remission and the changes in the pattern of bowel movement. Those, who record all items in the symptom diary for at least 10 days during the screening period and fulfill the eligible criteria will be randomized at a ratio of 1:1 into either the bifidobacterium quadruple viable tablet group or the placebo group for a 4-week the bifidobacterium quadruple viable tablet or placebo oral treatment. Visits will be scheduled at weeks 0-6 (or at discontinuation) to assess treatment efficacy, recurrence of IBS symptoms, and occurrence of adverse events. The time schedule and outcome assessment of the trial are presented in Figs. 1 and 2.

The protocol design is based on the Standard Protocol Items: Recommendations for Interventional Trials (SPIRIT) 2013 Checklist (Additional file 1). The overall supervision of our trial will be under the charge of the Clinical Trial Ethics Committee of Huazhong University of Science and Technology, any changes in the protocol will be submitted to and decided by the Ethics Committee. Subjects will receive timely 
medical treatment when adverse reactions occur during the trial, participants' health and safety will be the top priority. There will be sufficient time leaving for patients to consider whether to participate in the trial or not.

\section{Participants}

\section{Setting and recruitment}

A total of 300 patients diagnosed with IBS-D will be divided randomly into 2 groups (the bifidobacterium quadruple viable tablet group and placebo group) in a ratio of 1:1. The study is being conducted in the following fourteen hospitals: Wuhan Union Hospital, Peking Union Hospital, Chinese Academy of TCM Wangjing Hospital, Beijing Jishuitan Hospital, The Affiliated Sir Run Run Shaw Hospital of Zhejiang University School of Medicine, Zhejiang Provincial Hospital, Jilin University China-Japan Friendship Hospital, Jilin Provincial People's Hospital, The First People's Hospital Of Luoyang, The First People's Hospital Of Xinxiang, Lianyungang City Hospital, Jingzhou Chinese Hospital, Yan 'an University Affiliated Hospital, The Second Affiliated Hospital of Shandong University of Chinese Medicine. In all centers, physicians participated in this trial were informed of the admittance criteria, and participants will be recruited mainly via physician referrals from the gastroenterology clinics. There are posters and leaflets designed for the recruitment delivering to all centers and placing inside the hospital. Those who are willing to participant in this trial will need to contact the investigator and research assistants by telephone.

\section{Inclusion criteria}

1. Patients participated voluntarily and signed informed consent;

2. Aged 18-65 years old;

3. Patients were diagnosed as diarrhea predominant irritable bowel syndrome (IBS-D) by Rome IV criteria [23];

4. After two weeks of screening ( 7 days before the beginning of treatment), patients have abdominal pain at least 2 days a week, and the mean value of the most severe abdominal pain was more than 3 (11- NRS report). Meanwhile, the fecal traits were classified as type 6 or 7 at least 2 days;

5. During the screening period ( $14 \pm 3$ days before the onset of treatment), the patients completed the symptom diary for at least 10 days;

6. Patients discontinued the drugs used in the pre-screening stage for abdominal symptoms or diarrhea;

7. The lifestyle during the visit did not make any significant changes in the symptoms of diarrhea and abdominal pain (for example, starting a new diet, or changing the usual exercise pattern);

8. Colonoscopy report within two years of tertiary hospitals in China was normal or the researchers considered no clinical significance (such as chronic colitis diagnosed by congestion and edema, but 
no erosive ulcer; the diameter of colonic polyps was less than $3 \mathrm{~mm}$ and the number was less than 5).

\section{Exclusion criteria}

1. Having intestinal organic diseases, such as inflammatory bowel disease, intestinal tuberculosis, malabsorption syndrome, celiac disease, lactose intolerance, or other organic diseases;

2. Having history of abdominal and pelvic surgery, such as cholecystectomy (patients accepted appendectomy, patients who did not have intestinal complications after caesarean section, such as intestinal obstruction, and patients accepted endoscopic treatment of intestinal polyps can be included);

3. Having chronic pancreatitis, tumors, peptic ulcers, tuberculous peritonitis, chronic liver disease, liver cirrhosis and other digestive system non intestinal diseases (B ultrasonic diagnosis of chronic cholecystitis patients without typical biliary colic can be included);

4. Stool routine results were abnormal: fecal occult blood (+) or red blood cells (+) or white blood cells $(+)$;

5. Having other serious systemic diseases, including serious diseases such as heart, lung, kidney and other important organs, immunomodulatory diseases, metabolic diseases (diabetes, hyperthyroidism or hypothyroidism) or malignant tumors, and other diseases such as ovarian cysts and endometriosis;

6. Laboratory tests showed significant abnormalities; (1) hemoglobin < 1xLLN; (2) serum creatinine was more than $1 x U L N$, or creatinine clearance was less than $1 x L L N ;(3)$ abnormal liver function is defined as AST > 1.5xULN and / or ALT > 1.5xULN and / or total bilirubin > 1.5xULN;

7. Patients with severe mental illness or HAMD score > 20 points, or HAMA score > 29 points;

8. Abdominal pain occurs mainly at night during screening period, patients with unexplained emaciation, fever, jaundice, bloody stool or black stool, or BMI < 17;

9. Patients with a history of drug abuse or alcohol abuse (the abnormal adaptation caused by alcohol or drug use can lead to clinical morbid state, and at least 1 manifestations in 12 months: $\otimes$ Repeated use of drugs or alcohol to cause negligence or failure in work, academic or family responsibility, and $\nabla$ Use drugs or alcohol repeatedly on occasions where the body is in danger. $\nabla$ Legal problems related to the use of drugs or alcohol repeatedly. $\otimes$ Although drugs or alcohol cause social or interpersonal problems, they continue to use this substance.) and those who are allergic to the drug.

10. During the experiment, the accompanying drugs that affect gastrointestinal motility and function cannot be stopped, such as antibiotics, intestinal microecologic agents, proton pump inhibitors, etc.

11. Pregnant or breast-feeding women who plan to be pregnant during the trial period;

12. Less than 3 months after attending or completing other clinical trials;

13. Researchers think it is not suitable for the candidates.

\section{Randomization}


According to the randomization scheme of clinical research, statistical experts randomly code experimental drugs. The experimental drugs are randomly encoded as the unique identifier of the subjects. The subjects and investigators must stay in blind state from beginning to the end, changes of schedule will be communicated by the independent staff during the period. A block randomization model was applied with block size of 15 (20 patients in each block; 10 in the treatment group and 10 in the control group). A person not involved in this trial would perform the allocation.

1. Using the SAS 9.4 statistical software PROC PLAN process, a block randomization method will be used to generate a random arrangement of the subjects (the bifidobacterium quadruple viable tablet and placebo) which lists the random coding table.

2. Distribution of cases: The drug number assigned to each center will be random. In this trial, 14 centers are proposed, each center is based on the setting rules, that is, led by the principle investigator and sorted by the initial Chinese phonetic alphabet. The SAS software package will be used to generate the "central coded random numeric table" to obtain the random code of each center. Then drugs will be delivered to each center issued by code in sequence.

\section{Blinding}

This double-blind controlled trial is designed blinded to the investigators and subjects both. The blinding work will be accomplished by the primary sponsor, principal and statisticians. Two-dimensional blinding will be conducted. The first dimensional blindness will be implemented for drugs applying in the trial that the bifidobacterium quadruple viable tablet and placebo are in identical appearance and uniformly packaged, while the code of the experimental drug package will be set as the second dimensional blindness. Treatment allocations will be kept into envelops in confidence. One will be saved by staff member from Clinical Drug Trial Institution, while the others will be sent to each center with drugs as the emergency envelop.

\section{Study schedule}

\section{Informed consent acquirement}

Informed consent will be acquired before screening test. All patients recruited will be informed all contents involved in this trial in oral and written form both. There will be sufficient time for patients to consider whether to participate and raise any questions at their concern. And patients who are willing to participate will be required to sign informed consent paper.

\section{Screening period (day -14 to day $0 \pm 3$ days)}

Screening test will be conducted in this period to meet inclusion and exclusion criteria to determine if patients are admitted in this trial. Data of following contents will be collected:

1. General information including demographic characteristics(gender $\square$ age), previous and present medical history; 
2. Scores of Hamilton Anxiety and Depression Rating Scale (HAMA and HAMD);

3. Physical examination (general and abdominal) and vital signs including temperature, heart rate, respiratory rate and blood pressure;

4. Laboratory tests including blood routine, urine routine, stool routine and occult blood test, fasting blood glucose, tests of liver and kidney functions and electrocardiogram;

5. Abdominal ultrasonography and colorectal colonoscopy. Urine pregnancy test only in women of childbearing age;

6. Complete the symptoms log everyday including the strength of abdominal pain, fecal traits, the level of overall symptom relief, patterns of defecation, remedial medication and any other drugs history permitted in this trial;

7. Adverse events and concomitant medication.

Patients who are admitted in the trial will be allocated randomly into two groups in a ratio of 1:1, heading to the double-blind treatment period.

\section{Baseline (day 0)}

Before the treatment, subjects will be audited again if they meet admission criteria. Investigator shall give subjects physical exam again and subjects will be required to complete the IBS-QOL (quality of life) scale. Feces will be collected to study the microbiome and metabolome at this period.

\section{Treatment period (day 1 to day $28 \pm 3$ days)}

\section{Intervention}

Subjects of two groups will receive three tablets of either the bifidobacterium quadruple viable tablet or the matched placebo three times a day at 30 minutes after breakfast, lunch and dinner respectively for consecutive 4 weeks. During the treatment period, researchers will give subjects physical exams and record the results as well as vital signs. Subjects are supposed to keep completing the symptoms log every day and record remedial medication and any other drugs history permitted in this trial. HAMA, HAMD, IBS-QOL and laboratory tests will be updated at the end of treatment period.

\section{The bifidobacterium quadruple viable tablet and placebo}

Each bifidobacterium quadruple viable tablet contains a minimum of $0.5 \times 10^{6} \mathrm{cfu}$ Bifidobacterium infantis, Lactobacillus acidophilus and Enterococcus faecalis plus a minimum of $0.5 \times 10^{5} \mathrm{cfu}$ Bacillus cereus. The placebo tablet contains no active ingredients. The bifidobacterium quadruple viable tablets and the placebo tablets are identical in color, appearance and packaging. Both of them will be produced and provided by the Hangzhou Grand Biologic Pharmaceutical. INC. (Hangzhou, Zhejiang Province, China).

\section{Medical limitation}


During the trial, any drugs that may have impact on the evaluation of the effectiveness of bifidobacterium quadruple viable tablet affecting abdominal pain and diarrhea are not allowed (including antibiotics, parasympathetic inhibitors, muscle relaxants, opiates, other probiotics, antidepressants, anti-anxiety drugs and Chinese traditional medicine). Drugs have been used before baseline which are not opposite to the requirement of treatment or forbidden in exclusion criteria will be permitted during the trial.

\section{Withdraw criteria}

\section{Determined by investigator}

1. In cases of worsening disease or concomitant complications with a necessity of safety evaluation and curative treatment.

2. With forbidden therapy and medication history during the trial which will lead to bias for efficacy and safety evaluation.

\section{Participant request}

Patients will be allowed requesting to quit due to their priority, then their treatment will abort, regarded as shedding cases and recorded as "treatment failure". Reasons for withdraw will be followed and recorded in symptom diary while CRF of every patients will be saved for data analysis,

When subjects with relief of symptoms are unwilling to continue taking the treatment, they must not stop the medication without research's consent. And the 4-week follow-up will still be conducted after medication as planned.

\section{Following-up period (day 29 to day $42 \pm 3$ days)}

In this period, subjects shall update HAMA, HAMD and IBS-QOL again, and insist to complete the symptoms log and records of concomitant drugs and adverse events every day. Researchers shall perform physical exam and record the results and vital signs as same as previous times.

\section{Outcome assessment}

\section{Primary outcome measures}

The primary outcomes of this trial is the change of the strength of abdominal pain and the consistency of stool. The strength of abdominal pain will be evaluated by numerical rating scale using 0 to 10 to represent different levels of pain, while 0 means no pain and 10 means severe pain (unable to perform activities of daily living). The consistency of stool will be assessed by Bristol Stool Form Scale (BSFS), classified into seven categories:

Type 1: Separate hard lumps, like nuts (hard to pass)

Type 2: Sausage-shaped, but lumpy 
Type 3: Like a sausage but with cracks on its surface

Type 4: Like a sausage or snake, smooth and soft

Type 5: Soft blobs with clear cut edges (easy to pass)

Type 6: Fluffy pieces with ragged edges, a mushy stool

Type 7: Watery, no solid pieces, entirely liquid

The treatment will be considered effective when two criteria are reached simultaneously.

\section{Effective in improving abdominal pain for 4 weeks}

It will be regarded as remission of abdominal pain for a week when the average of the highest scores of abdominal pain everyday within the week after treatment decreases by more than $30 \%$ compared with the week before the treatment. And it will be regarded as the effectiveness of drugs in improving abdominal pain when the remission of abdominal pain lasts for more than half of the treatment period ( $\geq 2$ weeks).

\section{Effective in improving stool consistency for 4 weeks}

It will be regarded as improvement of stool consistency for a week when the number of days with Type 6 or 7 feces within the week after treatment decreases by more than $50 \%$ compared with screening period. And it will be regarded as the effectiveness of drugs in improving stool consistency when the improvement of stool consistency lasts for more than half of the treatment period ( $\geq 2$ weeks). In addition, a reduction of BSFS by 3 or more would be adopted as a quantitative indicator for assessing the improvement of stool consistency.

\section{Secondary outcome measures}

\section{Evaluation of overall symptom relief}

Using 1-7 to represent different levels of overall symptoms relief at last 7 days.

$1=$ complete remission

$2=$ obvious improvement

$3=$ slightly relief

$4=$ no change

$5=$ slightly worse

6= obvious exacerbation 
$7=$ the worst condition imaginable

\section{Frequency of defecation}

$1=$ once per day

$2=$ twice per day

$3=$ three times per day

4= more than three times per day

\section{Bloating (with borborygmus, increasing passing gas or belching frequently)}

$1=$ not at all

2= barely

$3=a$ little

4= moderate

$5=$ rather serious

$6=$ very serious

$7=$ unbearable

\section{Urgency of defecation}

$1=$ no urgency (feel no need to defecate when it comes to mind)

$2=$ slightly urgent (wish to defecate but not necessarily when it comes to mind)

$3=$ rather urgent (desire to defecate but not immediately)

$4=$ very urgent (have to rush to the bathroom at once)

\section{Use of remedial drugs}

Remedial medication will be allowed when subjects' symptoms aggravate to a health-threatening level evaluated by researchers in both groups. The decision of medication will be at the discretion of clinician for relieving abdominal symptoms and recorded in detail.

\section{Quality of life}


Subjects will be asked to finish IBS-QOL scale at baseline (week 0), the end of treatment period (week 4) and follow-up period (week 6) successively.

\section{Changes of microbiota and metabonomics}

Stool samples of subjects will be collected at baseline (week 0 ) and the end of treatment period (week 4) to study whether changes of microbiota and metabonomics occur after the treatment.

\section{Safety evaluation}

\section{Management of adverse events (AEs)}

Subjects will be instructed to report changes of their symptoms truthfully during the trial. Curative effects will be monitored, as well as adverse reactions and unexpected side effects. Adverse events must be recorded in detail whether relate to the trial or not, including time of occurrence, clinical manifestations, severity, duration, results of laboratory tests, management and outcome, follow-up times. History of medications is also significant to be on record to analyze causality between adverse events and experimental drugs. If any serious adverse event (SAE) occurs, it must be reported to the Province Food and Drug Administration, State Food and Drug Administration, primary sponsor institution, research institutions and the Ethics Committee within 24h. Record shall be signed and dated by the researcher who report serious adverse event. The blinding will be maintained until SAE occurs. Once blinding broken, the trial will be aborted and the subject will be marked as a shedding case. The management result will be reported to the supervisor with specific reason, date and sign recorded on CRF.

When any AE occurs, clinician shall evaluate subject's condition and provide suitable intervention or treatment. If any SAE occurs, the research institution must take necessary measures to keep subject out of health-threatening danger and stay follow-up until subjects have been resolved.

\section{Concern on laboratory tests}

Electrocardiogram examination and laboratory tests will be performed before and after the treatment for all subjects. Routine blood, urine, stool, and stool occult blood, fasting blood glucose, kidney function, and liver function tests will be included. Abnormal changes of results will be on concern and recorded within 24 hours after receiving reports, researchers will be supposed to judge whether there are onsets of adverse events. Re-examinations, causality assessments and necessary intervention will be at the discretion of clinicians.

\section{Causality assessment of adverse reaction (AR) and medication}

Once suspected adverse reactions occur, causality of ARs and medication will be assessed by items below:

1. Whether there is a reasonable time sequence between taking the medicine and the occurrence of ARs. 
2. Whether the suspected ARs are in accordance with known possible adverse reactions of the medicine.

3. Whether the suspected ARs can be explained according to patient's pathological conditions, concomitant medication, and treatment received at currently or past.

4. Whether the suspected ARs alleviate or relieve completely when reduce dosage or stop medication.

5. Whether the suspected ARs reoccur after receiving the same drug again.

\section{Data management and monitoring}

In this trial, we designed the paper CRF and built the EDC system (Electronic Data Capture System) according to the paper CRF, The investigator were responsible for collecting information from each of the subjects every follow-up, inputting to the EDC system and data reduction. The data manager is responsible for establishing the database which will only open access to investigator and supervisor to keep data and information confidentially. A training regarding the trial-related procedure will be conducted by principle investigator for all staff members participating before the implement of the trial. The data will be entered by the investigators or authorized persons (such as CRC, etc.), and the data manager will use EDC system for data management. eCRF will be provided for researchers and authorized persons to record the data. There will be CRCs who appointed by the primary sponsor responsible for periodic monitoring, checking subjects informed consent, screening and inclusion cases regularly in all research centers, as well as confirming:

1. All eCRFs are filled out correctly and consistent with original data;

2. All miss or omissions of data have been amended with researcher's sign and date;

3. For each subjects, changes of dosage of drugs or therapy, concomitant medication, withdraw and omission of examination have been confirmed and recorded;

4. Verify subjects withdrawing or dropping out of trial with explanation recorded on eCRF;

5. Confirm all AEs recorded, and SAEs must be reported and recorded with schedule time;

6. Confirm experimental drugs being supplied, stored, distributed and withdrew according to the regulation with corresponding records.

Database will be locked after the blind data verification report and statistical plan are finished, data will not be permitted to be modified anymore.

During the trial, experimental drugs and symptom diary will be delivered to patients in the ex-period and returned after the period to ensure the process will be conducted as good as expected. Patients will be supposed to record the code of drugs, date, dose, and time when take medication, which will be regarded as the indication of patients' compliance and included in analysis. Before every follow-up, investigators will make a phone call to patients to ensure if they are ready for the visit. Patients will also be able to contact investigators through the same method when they are confused or in emergency. Once there are 
patients withdrawing from the trial, the reasons for withdraw shall be recorded as soon as possible as well as all of the latest outcomes.

\section{Statistical methods}

Once the trial scheme is determined, a statistical analysis protocol will be worked out and performed by the principle investigator and specialized statisticians blinded to the treatment allocation.

\section{Sample size calculation}

Sample size calculation were performed by using PASS 13. The 4-week treatment effective rate would be adopted as the main evaluation indicator. According to the systematic review published by Yan Zhang et

al ${ }^{[24]}$, when regarded overall symptom relief as the main evaluation indicator, the effective rate of probiotics was $50 \%$ while placebo was $30 \%$ in IBS-intervened RCTs. On this basis and combined with clinical experience, it was assumed that the 4-week treatment effective rate of the experimental group was $50 \%$, and that of the placebo group was $30 \%$. In the case of $a=0.05$ and the power of test was $90 \%$ $(\beta=0.10)$, the required sample size would be at least 124 pairs. Considering the certain shedding and withdraw samples, the planned sample size would be 150 cases/group, and the total would be 300 cases (into a 1:1 ratio).

\section{Statistical analysis}

\section{General analysis}

Statistical analysis will be performed by using SAS 9.4 (or updated version) software (SAS Institute Inc, Cary, NC, USA). In terms of data description, quantitative data will be presented as mean \pm standard deviation, median, minimum, maximum, lower quartile (Q1), upper quartile (Q3), respectively. The categorical data will be presented as frequency and percentage. The comparison of the two groups will be analyzed in proper methods according to the type of indicators. The quantitative data from the two groups will be compared on the basis of data distribution (variance homogeneity, normal distribution) using group $t$ test or Wilcoxon rank-sum test. A chi-square test or the Fisher's exact test (if the chi-square test is not applicable) will be applied on the categorical data, and Wilcoxon rank-sum test or $\mathrm{CMH}-\chi 2$ test for the ranked data. Two-sided tests will be applied in all statistical tests, and a $\mathrm{P}$ value of equal to or less than 0.05 will be considered statistically significant. (except for special instructions).

The full analysis set (FAS), the per protocol set (PPS) and the safety set (SS) will be included for analysis. The FAS will be obtained on the basis of intention-to-treat (ITT) population, after exclusion from all randomized subjects who receive at least a single-dose treatment through a minimum and rational method. Last-observation-carried-forward will be applied for estimation of missing data. The PPS is defined as a set including all patients meeting eligibility criteria, finishing the treatment without major protocol deviations, with good compliance (between $80 \%-120 \%$ ) and completing the CRF as anticipated. 
The safety set will include those receive at least a single-dose treatment with data of safety evaluation. Carry-forward of missing data will not be in demand.

\section{Baseline characteristics}

The number of subjects and completions in each center and the shedding cases will be listed, as well as a detailed list of dataset sizes among groups, cases distributions in different centers, total shedding rates, and unfinished causes. There will be an elaborate description of the demographic characteristics (age, height, vital signs, etc.), medical history and medication history of the patients, and the age, height and weight of the two groups will be exhibited to measure the comparability.

\section{Compliance analysis}

Comparing the situation whether subjects takes the experimental medication on schedule and at the dosage, and whether they takes forbidden drugs and diet history between intervention and placebo group. Subjects with concomitant medication should be counted and listed in detail.

\section{Curative efficacy evaluation}

Baseline: Describe the baseline of each curative efficacy indicator.

Primary curative efficacy indicators: The treatment efficacy of the two groups will be described respectively. The comparison between the groups will be performed by using the $\mathrm{CMH}$ chi-square test which takes the central factors into consideration. If there is necessity in the correction of central and other factors, the Logistics regression model could be conducted depending upon the situation for comparison between groups.

Secondary curative efficacy indicators: The two groups were statistically described according to the categorical indicators including overall symptom relief evaluation, frequency of defecation, bloating (with borborygmus, increasing passing gas or belching frequently), urgency of defecation; The scores of quality of life (IBS-QOL scale) will be described as quantitative indicators. Comparison between groups will be conducted by general statistical methods above.

\section{Safety analysis}

Adverse events and adverse reactions in both groups will be required to be elaborated in a list, as well as frequencies and incidence of AEs, SAEs, ARs, and SARs. A series of physical examination, electrocardiogram and laboratory tests include blood routine, urine routine, stool routine and occult blood test, fasting blood glucose, tests of liver and kidney functions will be performed before and after the treatment. Re-examination may be required when necessary for assessing $\mathrm{AEs}$, and changes of the results of will be presented in a cross-tab.

\section{Protocol amendments}


Once this protocol has been approved by the Ethics Committee, a formal protocol amendment illustration will be required, and must be signed by the principal investigator and approved by the Ethics Committee again prior to correction and implement. The amendment shall be approved and signed by the sponsor after the modification. Any person participating in this trial shall not violate the protocol.

\section{Discussion}

Though probiotics are considered beneficial in IBS treatment according to several meta-analysis and systematical reviews ${ }^{[24-28]}$, specific probiotics recommendations for IBS management are still not possible yet for the lack of data from high-quality RCTs ${ }^{[13,29-31]}$. Most previous studies ignore the heterogeneity of IBS and lack of validated symptoms and QOL assessment, result in obscure outcomes of probiotics effectiveness. This trial aims at demonstrating the efficacy of the bifidobacterium quadruple viable tablet on patients with IBS-D by assessing symptom relief including abdominal pain, stool consistency, bloating, frequency and urgency of defecation. Non-gastrointestinal health status is also taken into consideration in the form of QOL, psychological status. In comparison with previous studies of probiotic products for IBS treatment, this multicenter collaborative study will have more rigorous methodology and quality assurance.

In addition, having been widely used in clinical practice for a long history, safety of probiotic preparations is still in concern for lack of assessment and systematic reporting of adverse events in probiotics intervention studies ${ }^{[32,33]}$. To fill the gap in probiotics application, this study also present outcome on safety assessment through monitoring adverse events, comprehensive physical examination, vital signs, biochemical tests and ECG which reflect patients' condition objectively. Health of patients is regarded as the priority and remedial measures are set up for the adverse events. Moreover, changes of microbiota and metabonomics in feces are also taken into consideration, drawing out the connection with symptom relief will be beneficial to gut microbiota researches.

In order to reduce bias, this study adopts adequate sample size, compliance analysis and conducted in multicenter throughout China to present the outcome in national level. Nevertheless, there are still limitations in this study. As IBS cannot yet be reliably monitored with biomarkers, subjective evaluation scales are adopted as outcome measures, which lack objective evidence and accuracy to verify the efficacy of given treatment. Diet habits vary significantly throughout China, it is difficult to standardize diets for patients from different area. So diet-related bias may not be controlled easily in such a largescale study.

As indicated by previous studies, effectiveness of stain- and dose-specific probiotics remain unclear [2931], we are expecting this trial will present the efficacy of bifidobacterium quadruple viable tablet in the IBS-D treatment, and the results will be considered as convictive high-quality evidence validating present paradigm.

\section{Trial status}


Participant recruitment is in preparation.

\section{Abbreviations}

IBS: Irritable bowel syndrome; IBS-D: Diarrhea predominant IBS; IBS-QOL: IBS-Quality of Life Questionnaire; HAMD: Hamilton Depression Rating Scale; HAMA: Hamilton Anxiety Rating Scale; BMl; ECG: Electrocardiogram; BSFS: Bristol Stool Form Scale; AE: Adverse events; SAE: Serious adverse event; AR: Adverse reaction; SAR: Serious adverse reaction; CRF: Case report form; EDC system: Electronic Data Capture System; CRC: Clinical research coordinator; RCT: Randomized control trial; FAS: Full analysis set; PPS: the per protocol set; SS: the safety set; ITT: intention-to-treat.

\section{Declaration}

\section{Ethics approval and consent to participate}

The study conforms to the principles of the Declaration of Helsinki (version Fortaleza 2013) ${ }^{[34]}$, and has been approved by the Clinical Trial Ethics Committee of Huazhong University of Science and Technology (the Approved No. of the Ethics Committee is 2018-137) and was registered at the Chinese Clinical Trial Registry (Chictr) platform on 10 August 2018 (Registration number: ChiCTR1800017721). The investigators must obtain written informed consent for participation, blood and stool samples collection from each participant before the trial.

\section{Consent for publication}

All authors have reviewed the final version of the manuscript and approved its publication.

\section{Availability of data and material}

The data will be available at the Research Manager (ResMan) platform (http://www.medresman.org/login.aspx) within 6 months after the completion of trial.

\section{Competing interests}

The authors declare that they have no competing interests.

\section{Funding}

No funding was received.

\section{Authors' contributions}


$\mathrm{XHH}$ is the principal investigator and takes responsibility for the study. XHH, TB, YQL, XQL, XHS, YL, ZHF and LZ participated in planning and designing the study and recruiting. HYZ contributed to manuscript writing. LLG supported data management and statistical analysis. TB was responsible for revising the manuscript. All authors have read and approved the final manuscript.

\section{Acknowledgements}

We gratefully acknowledge all the participants in this study. This work was undertaken with the support of the Hangzhou Grand Biologic Pharmaceutical. INC and fourteen hospitals involved as trial centers.

\section{Authors' details}

${ }^{1}$ Division of Gastroenterology, Union Hospital, Tongji Medical College, Huazhong University of Science and Technology, 1277 Jiefang Avenue, Wuhan, Hubei China.

${ }^{2}$ Division of Gastroenterology, Sir Run Run Shaw Hospital, Zhejiang University School of Medicine. 3 East Qingchun Road, Hangzhou, Zhejiang China.

${ }^{3}$ Division of Gastroenterology, Peking Union Medical College Hospital, No.1 Shuaifuyuan Wangfujing Dongcheng District, Beijing China.

${ }^{4}$ Division of Gastroenterology, Beijing Jishuitan Hospital, Xicheng District Xinjiekou No. 31 East Street.

${ }^{5}$ Peking University Clinical Research Institute, No.38 Xueyuan Road Haidian District, Beijing China.

${ }^{6}$ Hangzhou Grand Biologic Pharmaceutical. INC, 63 Jiuhuan Road, Jianggan District, Hangzhou, Zhejiang Province.

\section{References}

[1] Longstreth GF, Thompson WG, Chey WD, et al. Functional bowel disorder [J]. Gastroenterology, 2006, 130(5):1480-1491.

[2] [Accessed July 20, 2016]. http://theromefoundation.org/rome-iv/rome-iv-faqs/ Rome IV FAQs. February 2016:1-3. Rome Foundation.

[3] Sperber, A. D., Dumitrascu, D., Fukudo, S., Gerson, C., Ghoshal, U. C., Gwee, K. A., Whitehead, W. (2016). The global prevalence of IBS in adults remains elusive due to the heterogeneity of studies: a Rome Foundation working team literature review. Gut, 66(6), 1075-1082.

[4] Bai, T., Xia, J., Jiang, Y., Cao, H., Zhao, Y., Zhang, L., ... Hou, X. (2017). Comparison of the Rome IV and Rome III criteria for IBS diagnosis: A cross-sectional survey. Journal of Gastroenterology and Hepatology, 32(5), 1018-1025. 
[5] Wilkins T, Pepitone C, Alex B, Schade RR. Diagnosis and management of IBS in adults. Am Fam Physician 2012; 86: 419-426.

[6] Rivkin, A., \& Rybalov, S. (2016). Update on the Management of Diarrhea-Predominant Irritable Bowel Syndrome: Focus on Rifaximin and Eluxadoline. Pharmacotherapy: The Journal of Human Pharmacology and Drug Therapy, 36(3), 300-316.

[7] Collins, S. M. (2014). A role for the gut microbiota in IBS. Nature Reviews Gastroenterology \& Hepatology, 11(8), 497-505. doi:10.1038/nrgastro.2014.40

[8] Bhattarai, Y., Muniz Pedrogo, D. A., \& Kashyap, P. C. (2017). Irritable bowel syndrome: a gut microbiotarelated disorder? American Journal of Physiology-Gastrointestinal and Liver Physiology, 312(1), G52G62.

[9] Shanahan, F., \& Quigley, E. M. M. (2014). Manipulation of the Microbiota for Treatment of IBS and IBD -Challenges and Controversies. Gastroenterology, 146(6), 1554-1563.

[10] Harris, L. A., \& Baffy, N. (2017). Modulation of the gut microbiota: a focus on treatments for irritable bowel syndrome. Postgraduate Medicine, 129(8), 872-888.

[11] Zhuang, X., Xiong, L., Li, L., Li, M., \& Chen, M. (2017). Alterations of gut microbiota in patients with irritable bowel syndrome: A systematic review and meta-analysis. Journal of Gastroenterology and Hepatology, 32(1), 28-38.

[12] Dogan, B., Belcher-Timme, H. F., Dogan, E. I., Jiang, Z.-D., DuPont, H. L., Synder, N., ... Simpson, K. W. (2018). Evaluation of Escherichia coli pathotypes associated with Irritable Bowel Syndrome. FEMS Microbiology Letters.

[13] McKenzie Y.A., Thompson J., Gulia P. \& Lomer M.C.E. (2016) British Dietetic Association systematic review of systematic reviews and evidence-based practice guidelines for the use of probiotics in the management of irritable bowel syndrome in adults (2016 update). J Hum Nutr Diet. 29, 576-592.

[14] T. Wilkins and J. Sequoia, "Probiotics for gastrointestinal conditions: a summary of the evidence," American Family Physician, vol. 96, no. 3, pp. 170-178, 2017.

[15] Bifidobacterium quadruple viable tablet @ Instructions. Hangzhou Grand Biologic Pharmaceutical. INC. (in Chinese)

[16] Yuan, F., Ni, H., Asche, C. V., Kim, M., Walayat, S., \& Ren, J. (2017). Efficacy of Bifidobacterium infantis 35624 in patients with irritable bowel syndrome: a meta-analysis. Current Medical Research and Opinion, 33(7), 1191-1197.

[17] Lan, R., Koo, J., \& Kim, I. (2016). Effects of Lactobacillus acidophilus supplementation on growth performance, nutrient digestibility, fecal microbial and noxious gas emission in weaning pigs. Journal of 
the Science of Food and Agriculture, 97(4), 1310-1315.

[18] Wang S, Li H, Du C, Liu Q, Yang D, Chen L, Zhu Q, Wang Z. 2018. Effects of dietary supplementation with Lactobacillus acidophilus on the performance, intestinal physical barrier function, and the expression of NOD-like receptors in weaned piglets. PeerJ6:e6060.

[19] Liu, C., Zhu, Q., Chang, J., Yin, Q., Song, A., Li, Z., ... Lu, F. (2017). Effects of Lactobacillus casei and Enterococcus faecalis on growth performance, immune function and gut microbiota of suckling piglets. Archives of Animal Nutrition, 71(2), 120-133.

[20] Samli, H. E., Dezcan, S., Koc, F., Ozduven, M. L., Okur, A. A., \& Senkoylu, N. (2010). Effects of Enterococcus faecium supplementation and floor type on performance, morphology of erythrocytes and intestinal microbiota in broiler chickens. British Poultry Science, 51(4), 564-568.

[21] Duc L., Hong H., Barbosa T., Henriques A., Cutting S. (2004). Characterization of Bacillus probiotics available for human use. Appl. Environ. Microbiol. 70, 2161-2171. 10.1128/AEM.70.4.2161-2171.2004

[22] Bottone, E. J. (2010). Bacillus cereus, a Volatile Human Pathogen. Clinical Microbiology Reviews, 23(2), 382-398.

[23] Drossman DA. Functional gastrointestinal disorders: history, pathophysiology, clinical features and Rome IV. Gastroenterology. 2016. doi: https://doi.org/10.1053/j.gastro.2016.02.032.

[24] Zhang, Y., Li, L., Guo, C., Mu, D., Feng, B., Zuo, X., \& Li, Y. (2016). Effects of probiotic type, dose and treatment duration on irritable bowel syndrome diagnosed by Rome III criteria: a meta-analysis. BMC Gastroenterology, 16(1).

[25] Didari, T. (2015). Effectiveness of probiotics in irritable bowel syndrome: Updated systematic review with meta-analysis. World Journal of Gastroenterology, 21(10), 3072.

[26] Camilleri, M. (2018). Management Options for Irritable Bowel Syndrome. Mayo Clinic Proceedings, 93(12), 1858-1872.

[27] Barbara, G., Cremon, C., \& Azpiroz, F. (2018). Probiotics in irritable bowel syndrome: Where are we? Neurogastroenterology \& Motility, 30(12), e13513.

[28] Hungin, A. P. S., Mulligan, C., Pot, B., Whorwell, P., Agréus, L., ... Fracasso, P. (2013). Systematic review: probiotics in the management of lower gastrointestinal symptoms in clinical practice - an evidence-based international guide. Alimentary Pharmacology \& Therapeutics, 38(8), 864-886.

[29] Rondanelli, M., Faliva, M. A., Perna, S., Giacosa, A., Peroni, G., \& Castellazzi, A. M. (2017). Using probiotics in clinical practice: Where are we now? A review of existing meta-analyses. Gut Microbes, $8(6)$, 521-543. 
[30] Mazurak, N., Broelz, E., Storr, M., \& Enck, P. (2015). Probiotic Therapy of the Irritable Bowel Syndrome: Why Is the Evidence Still Poor and What Can Be Done About It? Journal of Neurogastroenterology and Motility, 21(4), 471-485.

[31] McFarland, L. V., Evans, C. T., \& Goldstein, E. J. C. (2018). Strain-Specificity and Disease-Specificity of Probiotic Efficacy: A Systematic Review and Meta-Analysis. Frontiers in Medicine, 5.

[32] Doron, S., \& Snydman, D. R. (2015). Risk and Safety of Probiotics. Clinical Infectious Diseases, 60(suppl_2), S129-S134.

[33] Wallace TC, MacKay D. The safety of probiotics: considerations following the 2011 U.S. Agency for Health Research and Quality report. J Nutr 2011;141:1923-4.

[34] WMA, World Medical Association. Declaration of Helsinki. J Am Med Assoc 2013; 227: 925-926.

\section{Additional File}

Additional file 1: Standard Protocol Items: Recommendations for Interventional Trial (SPIRIT) 2013 Checklist*. (DOC $122 \mathrm{~kb}$ )

\section{Figures}




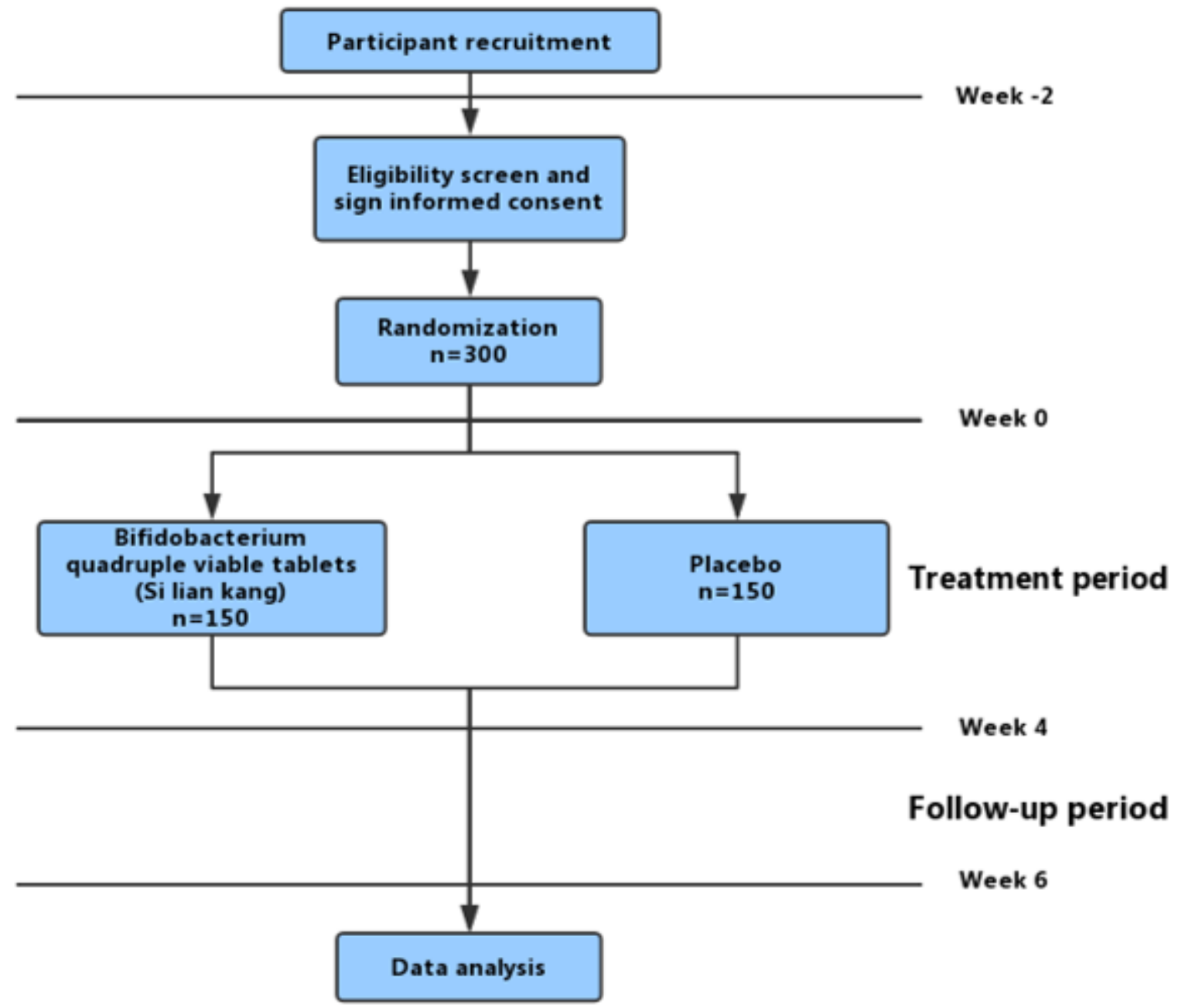

Figure 1

Flowchart of study. 


\begin{tabular}{|c|c|c|c|c|c|}
\hline & Screening & Baseline & Treatm & period & Follow-up period \\
\hline & -2 weeks & 0 week & 2 weeks & 4 weeks & 6 weeks \\
\hline \multicolumn{6}{|l|}{ Research activity } \\
\hline Sign informed consent & $\checkmark$ & & & & \\
\hline HAMA and HAMD & $\sqrt{ }$ & & & $\checkmark$ & $\sqrt{ }$ \\
\hline \multicolumn{6}{|l|}{ Eligibility screen } \\
\hline \multicolumn{6}{|l|}{ Intervention } \\
\hline \multicolumn{6}{|l|}{$\begin{array}{l}\text { Experimental drug } \\
\text { (Si lian kang) }\end{array}$} \\
\hline \multicolumn{6}{|l|}{ Placebo } \\
\hline \multicolumn{6}{|l|}{ Drug delivery } \\
\hline \multicolumn{6}{|l|}{ Drug withdraw } \\
\hline \multicolumn{6}{|l|}{ Data Collection } \\
\hline Socio-demographics & $\sqrt{ }$ & & & & \\
\hline Abdominal ultrasonography & $\sqrt{ }$ & & & & \\
\hline Colorectal colonoscopy & $\checkmark$ & & & & \\
\hline \multicolumn{6}{|l|}{ Outcome assessment } \\
\hline $\begin{array}{l}\text { Evaluation of abdominal pain } \\
\text { and stool traits }\end{array}$ & & $\sqrt{2}$ & $\sqrt{2}$ & $\sqrt{ }$ & $\sqrt{ }$ \\
\hline IBS-QOL & & $\sqrt{ }$ & & $\sqrt{ }$ & $\sqrt{ }$ \\
\hline \multicolumn{6}{|l|}{ Symptom diary delivery } \\
\hline Symptom diary withdraw & & & & & \\
\hline \multicolumn{6}{|l|}{ Safety assessment } \\
\hline Physical exam & $\sqrt{ }$ & $\sqrt{2}$ & $\sqrt{ }$ & $\sqrt{ }$ & $\sqrt{ }$ \\
\hline Vital signs & $\checkmark$ & $\sqrt{2}$ & $\sqrt{2}$ & $\sqrt{ }$ & $\sqrt{2}$ \\
\hline Laboratory tests and ECG & $\sqrt{2}$ & & & $\sqrt{2}$ & \\
\hline $\begin{array}{l}\text { Concomitant medication } \\
\text { history }\end{array}$ & & & & & \\
\hline Adverse events & $\leftarrow$ & & & & \\
\hline
\end{tabular}

Figure 2

Study schematic diagram. IBS irritable bowel syndrome, HAMA Hamilton Anxiety Scale, HAMD Hamilton Depression Scale, IBS-QOL IBS-Quality of Life Questionnaire, ECG electrocardiagram

\section{Supplementary Files}

This is a list of supplementary files associated with this preprint. Click to download.

- Additionalfile1.pdf 